\title{
AN EFFICIENT M-ARY QIM DATA HIDING ALGORITHM FOR THE APPLICATION TO IMAGE ERROR CONCEALMENT
}

\author{
Amit Phadikar ${ }^{1}$, Santi P. Maity ${ }^{2}$ \\ ${ }^{1}$ Department of Information Technology, MCKV Institute of Engineering, Liluah, \\ Howrah 711204, India. \\ amitphadikarerediffmail.com \\ ${ }^{2}$ Department of Information Technology, Bengal Engineering and Science University, \\ Shibpur, Howrah 711 103, India. \\ santipmaitydit.becs.ac.in
}

\begin{abstract}
Methods like edge directed interpolation and projection onto convex sets (POCS) that are widely used for image error concealment to produce better image quality are complex in nature and also time consuming. Moreover, those methods are not suitable for real time error concealment where the decoder may not have sufficient computation power or done in online. In this paper, we propose a data-hiding scheme for error concealment of digital image. Edge direction information of a block is extracted in the encoder and is embedded imperceptibly into the host media using quantization index modulation (QIM), thus reduces work load of the decoder. The system performance in term of fidelity and computational load is improved using M-ary data modulation based on near-orthogonal QIM. The decoder extracts the embedded features (edge information) and those features are then used for recovery of lost data. Experimental results duly support the effectiveness of the proposed scheme.
\end{abstract}

\section{KEY WORDS}

Error Concealment, Data Hiding, M-ary modulation, QIM, Interpolation.

\section{INTRODUCTION}

Rapid growth in computer power, as well as in wireless communication technology and the advent of the World Wide Web (WWW) make the Internet easily accessible to everyone. Hence, the use of multimedia signals increases lot during the last few years. At the same time, the imperfect transmission over wireless channel introduces vast amounts of random and burst errors leading to severe degradation in quality for the transmitted data due to fading, signal attenuations, and co-channel interference [1]. As a metre of fact, transmission of block-coded images through error prone wireless channels often results in lost blocks. Error concealment (EC) techniques, by exploiting inherent redundancy reduce visual artefacts through post processing at the decoder.

Several methods have been developed for recovery of lost data in the decoder, showing different trade-off between complexity and quality. A set of error concealment techniques that do not use data hiding but provide similar high level of performance have been proposed in the literature. Those methods are pixel domain interpolation (PDI) [2], directional interpolation (DI) $[3,4]$, projection onto convex sets (POCS) [5], block matching [6], neighbourhood regions partitioned matching [7] or spectral domain like discreet cosine transform (DCT) [8] and discreet wavelet transform (DWT) [9]. Li and Orchard provide a good review of these techniques and also propose a set of block-based sequential recovery techniques [10]. The above decoder side error concealment schemes [2-10] are mainly based on two steps. First, the 
decoder estimates some features of lost information like edge direction information or motion vector. Second, the decoder interpolates lost information from the estimated features in spatial, transform or temporal domain. These schemes work well in simplified loss scenarios where successfully received data are assumed to be reconstructed as loss-free. However, this is most often not the case depicting the real scenario. Moreover, the first step suffers from higher computational complexity and also takes large time. In literature, it is reported that the first step takes roughly $30 \%$ of total computation load. As a result, those schemes are not suitable for real time error concealment where the decoders may not have sufficient computation power or done in online.

To overcome those problems Liu and $\mathrm{Li}$ [11] are the first authors who use data hiding as an error concealment tool. The scheme extracts the important information of an image, like the DC components of each $(8 \times 8)$ block, and embeds it into the host image to achieve the goal. Adsumilli et al. [12] propose a robust image error concealment algorithm using watermarking. The scheme embeds a low-resolution version of each image or video frame into itself using spread-spectrum modulation. Authors analyze various advantages and disadvantages of the full frame discrete cosine transform (DCT) against the conventional block-based DCT embedding. Gur et al. [13] investigate an error concealment technique of image using data hiding. The scheme embeds macro block-based, best neighbourhood matching (BNM) information into the original image in DWT domain. The experimental results indicate that the above error concealment technique is a promising one, especially for the erroneous channels causing a wider range of packet losses. Recently, Phadikar et al. [14] propose an image error concealment scheme using dual-tree complex wavelet transform (DTCWT) and quantization index modulation (QIM). The experimental results indicate that the inherent redundancy in DTCWT provides better error concealment over traditional DWT with an increase in complexity due to DTCWT. Yýlmaz et al. [15] and Yin et al. [16] propose a data hiding that embeds edge direction information into the host image. So the decoder needs only to perform the second step using the features (edge direction information) that are already extracted by the encoder. In addition, performing feature extraction at the encoder is more effective as the encoder usually have access to more information of the data. Though the complexity of the encoder is increased, the encoder usually has more computational resources and often can perform the tasks off-line. The main drawback of those schemes $[15,16]$ is fragile in nature, as those schemes use even-odd embedding. As a result, the extracted edge information may be wrong due to slight signal modification. In our scheme, we overcome this problem using M-ary data modulation based on near-orthogonal QIM.

This paper proposes an error concealment scheme for digital images using data hiding. The goal is achieved by embedding some important information like edge direction information of each block extracted from the original image itself. The edge information is embedded in the DCT domain. We have select DCT domain as in reality $80 \%$ images are till JPEG compressed form. The advantage of using DCT for data hiding based error concealment is the extensive study of human visual system (HVS) in this domain, which has resulted in the standard JPEG quantization table. As a result, the scheme can be more adaptive to HVS. The major contributions of this paper are two fold. First, a QIM based data hiding is used to embed edge direction information of a block at the encoder. This helps decoder to extract correct edge direction information in an easy way and also in less time. In other words, this decreases the workload at the decoder. Moreover, QIM is selected as it provides considerable performance advantages over spread-spectrum and low-bit(s) modulation in terms of the achievable performance trade-offs among distortion, rate, and robustness of the embedding [17]. Second, the system performance in terms of fidelity and robustness is improved using M-ary data modulation that is implemented using near-orthogonal QIM. To the best of our knowledge Mary QIM has not yet been explored by watermarking research community in image error concealment applications.

The rest of the paper is organized as follows: Problem formation is outlined in Section 2. Basic principles and key features of M-ary near-orthogonal QIM are outlined in Section 3. The 
proposed error concealment scheme is introduced in Section 4. Some experimental results are presented in Section 5. The paper is concluded in Section 6.

\section{PROBLEM FORMATIONS}

In an image, the nearby pixel values are highly correlated that makes it possible to recover the information of the lost block by spatial-domain interpolation. In this section, we discuss the interpolation technique for the recovery of both smooth and edge blocks.

\subsection{Smooth Block Concealment}

If a lost block is a non-edge block, each pixel of a lost block is calculated as a linear combination of the nearest pixels in the block boundaries. In this method, pixel values of the lost block are interpolated by the following formula [3]:

$$
p(r, c)=\frac{d_{R} p_{L}(c, 1)+d_{L} p_{R}(c, S)+d_{B} p_{T}(1, i)+d_{T} p_{B}(S, r)}{d_{L}+d_{R}+d_{T}+d_{B}}
$$

where $p(r, c)$ is the interpolated pixel value, and $p_{x}(r, c)$ represents values of the nearest pixels of the four neighbouring blocks like top (T), bottom (B), left (L), right (R). The symbol $d_{x}$, is the distance between $p(r, c)$ and $p_{x}(r, c)$. We have used $\mathrm{p}(1,1)$ to represent the left bottom corner of an $(S \times S)$ macro block. The first and second coordinate presents the horizontal and vertical direction, respectively.

\subsection{Edge Block Concealment}

Since edge integrity plays an important role in human visual perception, we can utilize spatially correlated information more thoroughly by performing interpolation, based on edge direction in the local neighbourhood of the lost block. Then the recovery of lost block consists of two steps i.e. finding edge direction information and then edge directed interpolation.

\subsubsection{Classification of Edge Direction by Gradient Voting}

An efficient and widely used method for edge detection is calculating the gradient field by means of the mask. In the present scheme, for simplicity we only consider four types of edges and one block contains only one type of edge. Let R1, R2, R3, and R4 denote the responses of the mask in Fig. 1, from left to right, where R's are given by Eq. 2. Suppose that the four masks are run individually through an image. If at a certain point in the image, $\left|R_{i}\right|>\left|R_{j}\right|$ for all $i \neq j$, the corresponding point is said to be more likely associated with a line in the mask $i$. For example, if a point in the image, $\left|R_{i}\right|>\left|R_{j}\right|$ for $\mathrm{j}=2,3,4$ that particular point is said to be more likely associated with a horizontal line [18].

$$
R=w_{1} z_{1}+w_{2} z_{2}+\ldots+w_{9} z_{9}
$$

where $z_{i}$ is the gray value of the pixel associated with mask coefficients $w_{i}$.

\begin{tabular}{|c|c|c|}
\hline-1 & -1 & -1 \\
\hline 0 & 0 & 0 \\
\hline 1 & 1 & 1 \\
\hline
\end{tabular}

(a)

\begin{tabular}{|c|c|c|}
\hline-1 & -1 & 0 \\
\hline-1 & 0 & 1 \\
\hline 0 & 1 & 1 \\
\hline
\end{tabular}

(b)

\begin{tabular}{|c|c|c|}
\hline-1 & 0 & 1 \\
\hline-1 & 0 & 1 \\
\hline-1 & 0 & 1 \\
\hline
\end{tabular}

(c)

\begin{tabular}{|c|c|c|}
\hline 0 & -1 & -1 \\
\hline 1 & 0 & -1 \\
\hline 1 & 1 & 0 \\
\hline
\end{tabular}

(d)

Figure 1. Edge detection mask: (a) Horizontal, (b) $+45^{0}$, (c) vertical, (d) $-45^{0}$. 


\subsubsection{Edge Directed Interpolation}

For the selected direction, a series of one-dimensional linear interpolations are carried out to obtain the pixel values of the lost block. Interpolated pixel values within the lost block are calculated by the following simple formula [3]:

$$
p(r, c)=\frac{d_{1} p_{2}+d_{2} p_{1}}{d_{1}+d_{2}}
$$

where $p_{1}$ and $p_{2}$ are the points in boundaries from which the missing pixel is interpolated. Symbols $d_{1}$ and $d_{2}$ denote the distance of $p(r, c)$ from $p_{1}$ and $p_{2}$, respectively.

\section{BASIC PRINCIPLES AND KEY FEATURES OF M-ARY QIM}

The basic quantization index modulation (QIM) algorithm quantizes feature vector $X$, using a quantizer $Q_{\Delta}($.$) which chosen from a family of quantizer based on the message bit (m)$ to be embedded [17]. The watermarked featured vector $\tilde{X}$ is then obtained as follows:

$$
\tilde{X}=Q_{\Delta}(X+d(m))-d(m) ; m \in\{0,1\}
$$

where $\Delta$ is a fixed quantization step size, $d($.) is the dither used for embedding watermark bit.

At the decoder, the test signal $\tilde{X}$ is requantized using the same family of quantizer to determine the embedded message bit i.e.

$$
\hat{m}=\arg \min _{m \in\{0,1\}} \sum_{i=1}^{L}\left|\tilde{X}-Q_{\Delta}(\tilde{X}+d(m))-d(m)\right|
$$

where we have an assumption that the scheme uses a soft decoder and the symbol ' $L$ ' is the length of the dither. The performance of the traditional QIM can be further improved by M-ary modulation. We have modified the traditional QIM [17] to make it an M-ary modulation and is described in the next subsection.

\subsection{M-ary QIM}

In M-ary modulation, a group of symbols are treated as a single entity. We can relate the number of bits $(N)$ and the number of different symbols $(M)$ with the following equation [19]:

$$
M=2^{N}
$$

Obviously, the binary transmission system is a special case of an M-ary data transmission system. An effective method to implement an M-ary modulation technique is based on nearorthogonal dither. A group of near-orthogonal dither $d_{i} \in\left\{d_{0}, d_{1}, \ldots, d_{M-1}\right\}$ are generated independently using Hadamard function of standard math library and random number generated using a secret key (k). The smallest possible Hadamard matrix is of order two and is defined as:

$$
H_{2}=\left[\begin{array}{cc}
1 & 1 \\
1 & -1
\end{array}\right]
$$

The Hadamard matrix with an order of power in two may be constructed from $\mathrm{H}_{2}$ based on Kronecker product and is given by:

$$
H_{L}=H_{2} \otimes H_{L / 2}=\left[\begin{array}{cc}
H_{L / 2} & H_{L / 2} \\
H_{L / 2} & -H_{L / 2}
\end{array}\right]
$$


The basic characteristic of the Hadamard matrix is that all rows and column are orthogonal. The sequence of random number is an i.i.d., following Gaussian distribution $\eta(0,1)$. The generation of dither can be defined as follows:

$$
d_{i}=H(.) \times R \times \Delta / 2
$$

where $H($.$) is the Hadamard function to generate orthogonal code, \Delta$ is the step size for dither modulation and $R$ is a random number that is generated using following rule:

$$
R=\left[\Re_{L}\left(k_{1}\right)+\Re_{L}\left(k_{2}\right)+\cdots+\Re_{L}\left(k_{n}\right)\right] / n
$$

The function $\Re(k)$ is the random number generator. The symbol $R$ is multiplied with $H($.$) to$ increase security of the system.

One of the prominent characteristic of dither generated in this manner is their nearorthogonal property [19] i.e.

- $d_{i}$ for $\mathrm{i}=1,2 \ldots, M$ should be distinct sequences.

- The spatial correlation $\left\langle d_{i}, d_{j}\right\rangle, i \neq j$ should be minimum. Ideally, sequence $d_{i}$ and $d_{j}$ should be orthogonal when $i \neq j$.

Those properties are illustrated by Fig. 2, where as an example $d$ is a set of dithers with 256 elements and the correlations of $d_{50}$ with all other dither in the set are shown.

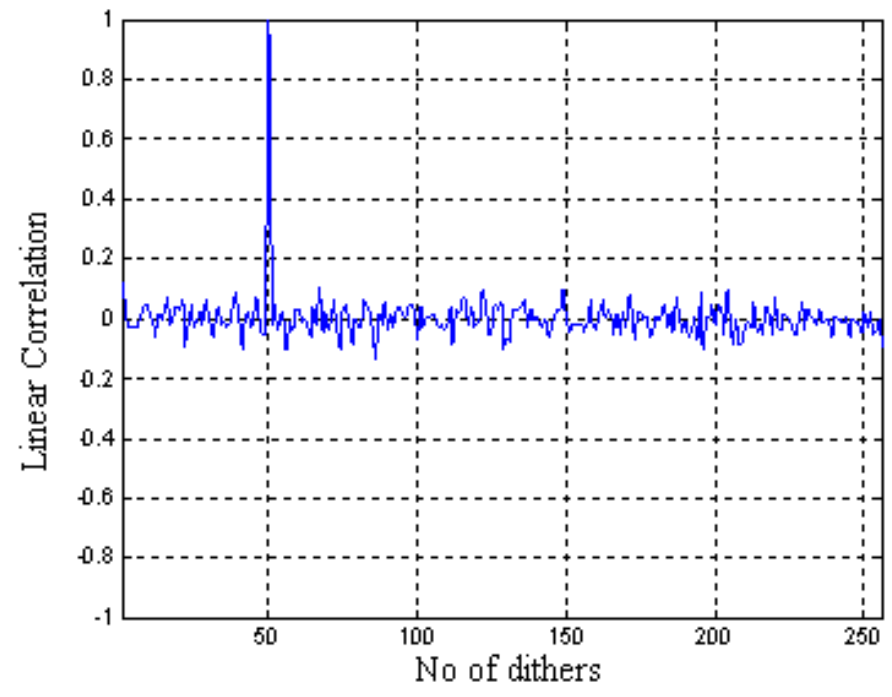

Figure 2. Linear correlation between a dither and its near-orthogonal version.

If each dither ' $d$ ' in the group is used to represent an M-ary message symbol $m \in\{0,1, \ldots, M-1\}$, it consists of $\log _{2} M=N$ bits of information once chosen for data embedding. With a QIM embedding function, the message ' $m$ ' can be embedded into the feature vector $(X)$ according to the following rule:

$$
\tilde{X}=Q_{\Delta}(X+d(m))-d(m) ; m \in\{0,1, \ldots, M-1\}
$$

For watermark decoding, the same set of dither $d \in\left\{d_{0}, d_{1}, \ldots, d_{M-1}\right\}$ is used. Fig. 3 shows the block diagram of watermark decoding in M-ary system. At the decoder, the test signal $\tilde{X}$ is requantized using the family of quantizer ' $d_{i}$ ' to determine the embedded message bit i.e. 


$$
\hat{m}=\arg \min _{m \in\{0,1, \ldots, M-1\}} \sum_{i=1}^{L}\left|\tilde{X}-Q_{\Delta}(\tilde{X}+d(m))-d(m)\right|
$$

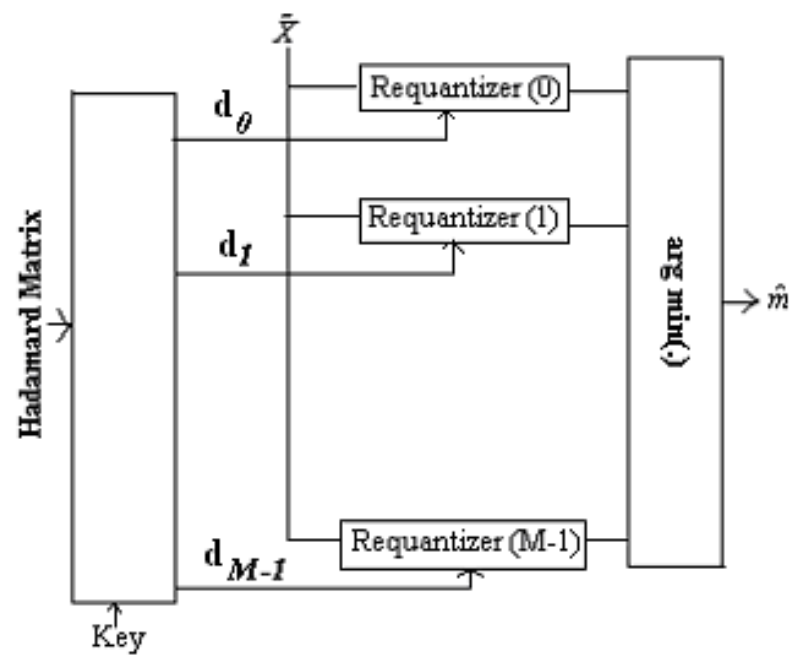

Figure 3. Structure of decoder for the extraction of $M$-ary watermark.

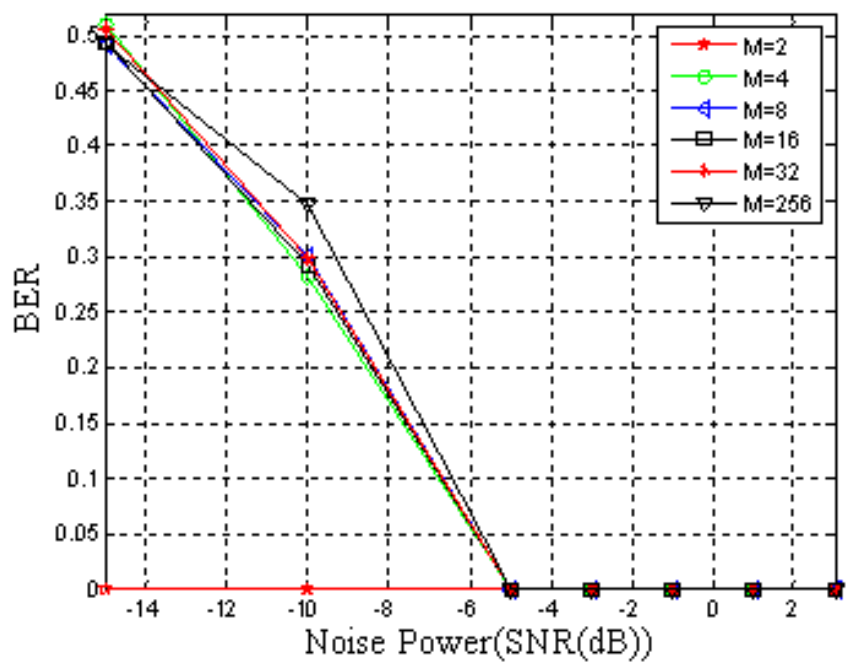

Figure 4. Bit error rate (BER) performance for different noise power. Number of signal point:

65536, watermark power: $9.2 \mathrm{~dB} \mathrm{~dB}$, capacity: 4096.

In the case of a conventional M-ary decoder illustrated in Fig. 3, the total number of operations required for the decoding of an M-ary symbol is approximately,

$$
T_{0}=L M
$$

where $L$ is the length of the feature vector. One operation is defined as one real division, one real addition plus one real subtraction. Apparently, $T_{0}$ is a linear function of $\mathrm{M}$. So for larges value of $M>4$ the computational workload for data extraction advances exponentially i.e. $O\left(2^{N}\right)$. In other words, the greater value of $M$, the better system performance in term of robustness for a fixed capacity, with the increase in decoding complexity. Fortunately, M-ary modulation does not increase any computation complexity in our scheme. In our scheme, the edge information is represented by five symbols. As a result, the proposed scheme does five correlation calculation for detecting a symbol, while traditional QIM needs six correlation calculation for detecting the 
edge type. Fig. 4 highlights robustness performance in term of bit error rate (BER) for different $M$ values. The word BER here implies the error in the extracted watermark signal when the watermarked signal is transmitted through the noisy channel or manipulation is done by some user. The BER is calculated through simulation by transmitting image data through noisy channel. It is seen that even if the noise power reaches to $-5 \mathrm{~dB}$ there is no BER even for large $\mathrm{M}$ values. Each point on the curves is obtained as the average value of 100 independent experimentations conducted over large number of benchmark images.

\section{PROPOSED ERROR CONCEALMENT TECHNIQUE}

The overall error concealment scheme consists of two stages: i.e. image encoding and image decoding process. The detailed block diagram representations of encoding and decoding process are shown in Fig. 5 and Fig. 6, respectively.

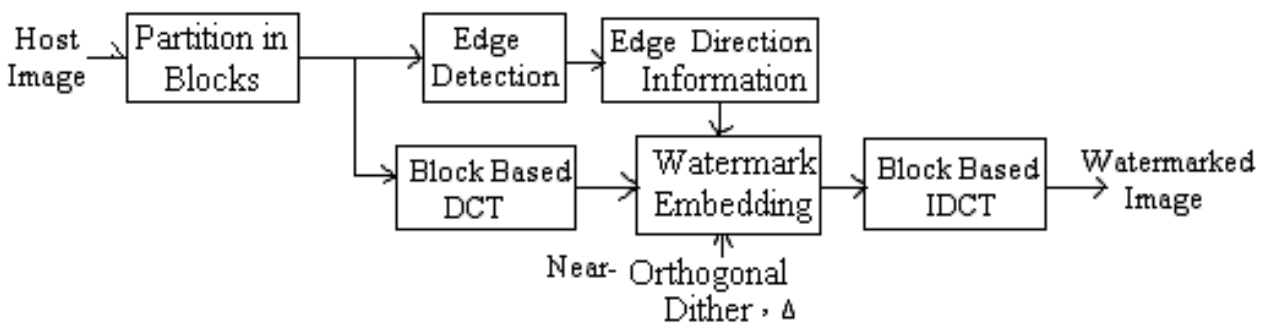

Figure 5. Block diagram of error concealment encoding process: Encoder.

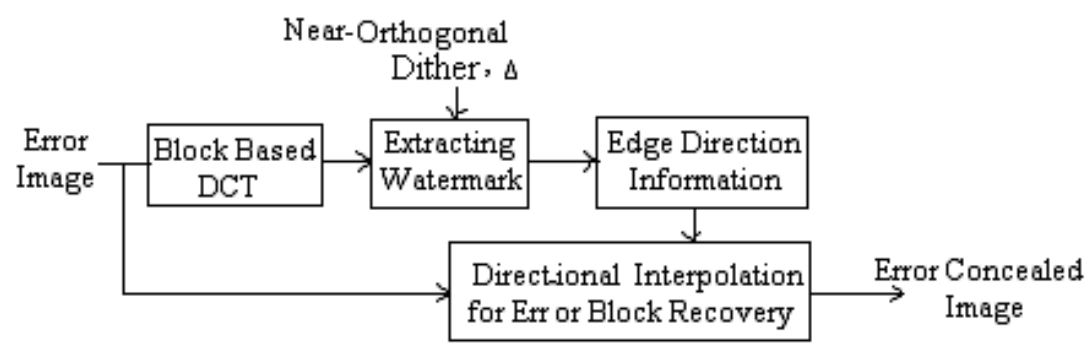

Figure 6. Block diagram of error concealment decoding process: Decoder.

\subsection{Image Encoding Process}

The objective of image encoding process is the extraction of edge information followed by embedding of that edge information at the encoder using M-ary modulation. The inputs to the encoding process are the gray scale image and secret keys for the generation of near-orthogonal dithers that are used for watermark bits embedding. The image encoding process consists of the following steps:

Step 1:Block Based Partitioning of Image: The original (host) image $(\mathrm{H})$ is partitioned into nonoverlapping blocks of size $(S \times S)$. We select $S=8$ for watermark insertion, as the effect of JPEG compression affects independently the each bit embedded in the block.

Step 2: Extraction of Edge Information: Various edge directional operators/masks are applied on each block and the blocks are categorized as non-edge or edge block depending on the response of the operators. If a block contains an edge, the type of edge direction is determined as described in Section 2.2.1 [18].

Step 3: Representation of Edge Information: The edge information of each block is represented by ' $m$ ' bits symbol depending on the type of edge it contains. Table 1 shows a typical such representation when types of various directions of edges are considered to be of four types. The 
MSB (most significant bit) represents whether the block contains an edge or not. If the MSB is zero, it then signifies that the block contains no edge. The next two bits represent the type of edge direction. Table 1 shows a set of five symbols by which respective edge information of a block is represented. In other words, five dithers are sufficient for embedding edge information of a block using M-ary modulation.

Table 1. Symbolic representation of edge information for a block.

\begin{tabular}{|c|c|c|c|}
\hline Bit 2 $^{\text {nd }}$ & Bit 1 $^{\text {st }}$ & Bit 0 $^{\text {th }}$ & Significance (Edge Type) \\
\hline 0 & 0 & 0 & No edge \\
\hline 1 & 0 & 0 & Horizontal \\
\hline 1 & 0 & 1 & $+45^{0}$ \\
\hline 1 & 1 & 0 & Vertical \\
\hline 1 & 1 & 1 & $-45^{0}$ \\
\hline
\end{tabular}

Step 4: Image Transformation: The 2-dimensional DCT of each cover image block is calculated.

Step 5: Watermark Embedding: The edge orientation information of each block is represented by a symbol $(\mathrm{m})$ as described in Step 3 and the information/message $(\mathrm{m})$ is embedded as watermark into another block by modulating DCT coefficients based on M-ary modulation. The different steps for data embedding are described as follows:

A): Generation of near-Orthogonal Dither:

Depending on the number of symbols (M), a group of near-orthogonal dithers $d_{i} \in\left\{d_{0}, d_{1}, \ldots, d_{M-1}\right\}$ are generated independently using Hadamard function of standard math library and a secret key $k$ as described in Eq. (9). The length of $d_{i}$ is equal to the number of coefficients that are used for embedding watermark bit in a block. In our scheme, one symbol (m) is represented by 3 bits and the length of $d_{i}$ is of 63 , as one $(8 \times 8)$ block contains $63 \mathrm{AC}$ coefficients. The scheme leaves DC coefficients unchanged at the time of data embedding, as use of DC component for watermark embedding reduces image fidelity of the watermarked image.

$B)$ : Watermark bit Insertion: The message $(m)$ is embedded in a block by modulating AC coefficients. The $\mathrm{q}^{\text {th }}$ watermarked AC coefficient $S^{q}$ is obtained as follows:

$$
S^{q}=Q_{\Delta}\left\{X^{q}+d(m)\right\}-d(m) ; m \in\{0,1, \ldots, M-1\}
$$

where $X^{q}$ is the original $q^{\text {th }}$ AC coefficient, $Q$ is a uniform quantizer (and dequantizer) with step $\Delta$. The symbol $d(m)$ is the dither that is used to represent a message/symbol $(m)$ for watermark embedding. As an alternative of M-ary modulation, one can also embeds 3 bits in an $(8 \times 8)$ block by dividing the AC coefficients into three non-overlapping sets, where one set can be used for embedding one watermark bit. But, this alternative data embedding process will make the scheme more fragile in nature. So, M-ary modulation is the appropriate choice for embedding 3 bits in a block. After watermark embedding, the inverse DCT is performed and the watermarked image is obtained.

\subsection{Image Decoding Process}

The proposed error concealment scheme is blind in the sense that the decoding process does not require the original/host image. In exigency of some blocks of the received signal $\left(\mathrm{H}^{\prime}\right)$ that are lost during transmission, are concealed at the receiver using different steps as described follows. 
Step 1: Steps 1, 4, and 5(A) of image encoding process are performed, over the received or possibly damaged image. The same step size $(\Delta)$ is used at the time of decoding that was used at the time of encoding. In order to achieve successful error concealment, the exact location of the error, i.e. damaged blocks, should be detected using some sophisticated error detection mechanisms. In our scheme the differences of pixel values between two neighbour lines are used for detecting transmission errors [20].

Step 2: Watermark Extraction: The edge orientation information of a lost block is extracted from the block that is used at the time of embedding. The message $(m)$ is extracted from the received DCT coefficients of a block using the following rule.

$$
\hat{m}=\arg \min _{m \in\{0,1, ., M-l\}} \sum_{i=1}^{L}\left|\tilde{X}-Q_{\Delta}(\tilde{X}+d(m))-d(m)\right| ;
$$

The extracted watermark information $\hat{m}$ represents the information about the edge direction of a block. It is noted that though our scheme uses M-ary modulation, the decoding complexity till less than the traditional QIM. Using traditional QIM one has to do six correlations calculations (for one watermark bit two dithers are needed. So for three watermark bits it calculates six correlations, as edge type of a block is represented by 3 bits) for detecting edge type. In M-ary modulation, it only takes five correlations as five symbols are sufficient to represent the edge type of a block.

Step 3: Recovery of Lost Blocks (Error Concealment): If a block is lost, the extracted edge direction information $(\hat{m})$ for that block is then used for the concealment purpose. If the lost block is a smooth block, recovery is done as described in Section 2.1. On the other hand, if the lost block is an edge block, the recovery is done by edge directed interpolation as described in Section 2.2.2.

\section{PERFORMANCE EVALUATIONS}

This section presents simulation results to show performance of the proposed error concealment technique. The efficiency of the proposed technique is evaluated over a large number of benchmark images [21] including eight popular test images: Lena, Fishing boat, Pepper, Cameraman, Baboon, Opera, Pueblo bonito, F16 shown in Fig. 7. The image database contains 29 test images having varied image characterises. All of the test images are $8 \mathrm{bit} / \mathrm{pixel}$ gray scale images with size $(512 \times 512)$. The value of step size $(\Delta)$ taken into consideration is 8 (i.e. watermark power (WP) of $7.27 \mathrm{~dB}$ ). In this study, we use peak-signal-to-noise-ratio (PSNR dB) and mean-structure-similarity-index-measure (MSSIM) [22] as the objective measures of the watermarked image quality. PSNR $(\mathrm{dB})$ is defined as follows:

$$
P S N R(\text { in } d B)=10 \log _{10}\left[\frac{255^{2}}{\frac{1}{X \times Y} \sum_{r=1 c=1}^{X} \sum_{c=1}^{Y}[P(r, c)-\tilde{P}(r, c)]^{2}}\right]
$$

where $P(r, c)$ represents the pixel value at coordinate $(r, c)$ in the original undistorted image, and $\tilde{P}(r, c)$ represents the pixel value in the watermarked (stego) image. $\mathrm{X}$ and $\mathrm{Y}$ are the number of rows and columns, respectively. MSSIM is defined as follows:

$$
\operatorname{MSSIM}(P, \bar{P})=\frac{1}{M^{\prime}} \sum_{j^{\prime}=1}^{M^{\prime}} \operatorname{SSIM}\left(P_{j^{\prime}}, \bar{P}_{j^{\prime}}\right)
$$

where

$$
\operatorname{SSIM}(P, \bar{P})=[l(P, \bar{P})]^{\delta} \cdot[c(P, \bar{P})]^{\beta} \cdot[s(P, \bar{P})]^{\gamma}
$$


The function $l(P, \bar{P}), c(P, \bar{P})$ and $s(P, \bar{P})$ are the luminance comparison, contrast comparison and structure comparison function, respectively. The symbol $\delta>0, \beta>0$ and $\gamma>0$ are the parameters used to adjust the relative importance of the components. Note that $0 \leq \operatorname{MSSIM}(P, \bar{P}) \leq 1$, and $\operatorname{MSSIM}(P, \bar{P})=1$ corresponds to no distortion. The symbols $P_{j^{\prime}}$ and $\bar{P}_{j^{\prime}}$ are the image contents at the $j^{\prime}-t h$ local window, i.e. the block, and $M^{\prime}$ is the number of samples in the quality map.

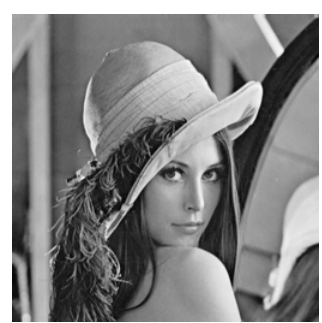

(a)

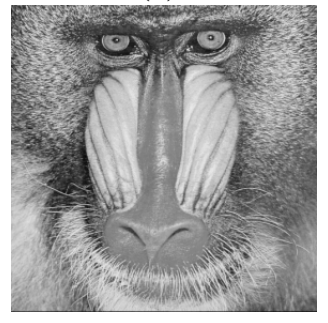

(e)

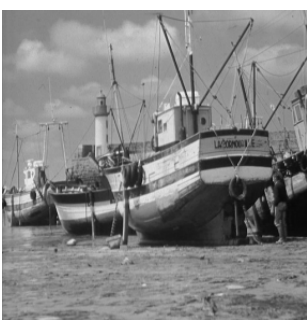

(b)

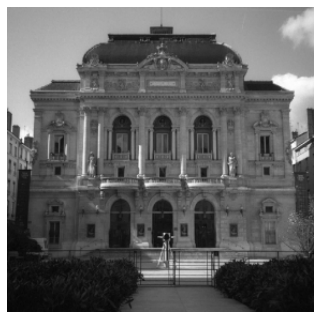

(f)

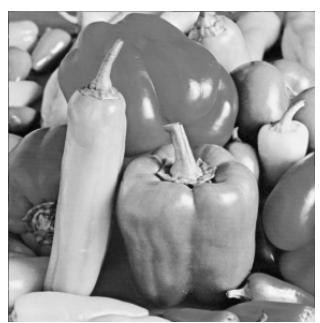

(c)

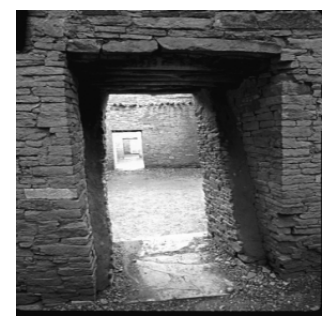

(g)

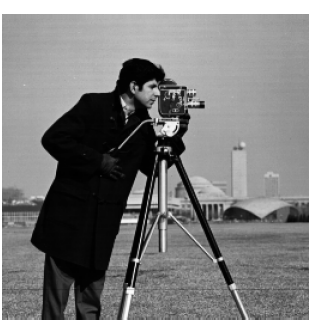

(d)

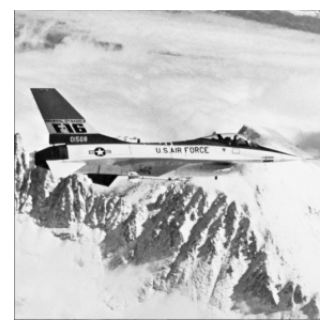

(h)

Figure 7. Test images; (a): Lena, (b): Fishing boat, (c): Pepper, (d): Cameraman, (e): Baboon, (f): Opera, (g): Pueblo bonito, (h): F16.

In the first set of experiments, we have studied the performance of the proposed scheme in term of fidelity of the watermarked image. Table 2 illustrates the PSNR (dB) and MSSIM values of various watermarked images after embedding edge information to the respective host images. Fig. 8 shows the watermarked image visually. The length of the watermark is 12288 bits that are used to represent the edge information of all $(8 \times 8)$ blocks of an image of size $(512 \times 512)$. From Table 2 it is clear that the quality of the watermarked image in term of PSNR $(\mathrm{dB})$ is almost same for various test images. It is also interesting to see from the results of Table 2 that though for a given watermark power the visual quality in PSNR $(\mathrm{dB})$ for the watermarked image does not vary much but high value of structural similarity is found for the image having high texture image like Baboon and Pueblo bonito.

Table 2. PSNR (dB) and MSSIM of the watermarked image.

\begin{tabular}{|c|c|c|c|c|}
\hline Images & Lena & Fishing boat & Pepper & Cameraman \\
\hline PSNR (dB) & 40.88 & 40.66 & 40.88 & 40.77 \\
\hline MSSIM & 096 & 0.97 & 0.96 & 0.95 \\
\hline & Baboon & Opera & Pueblo bonito & F16 \\
\hline PSNR (dB) & 40.93 & 40.91 & 40.87 & 40.80 \\
\hline MSSIM & 0.98 & 0.97 & 0.98 & 0.95 \\
\hline
\end{tabular}




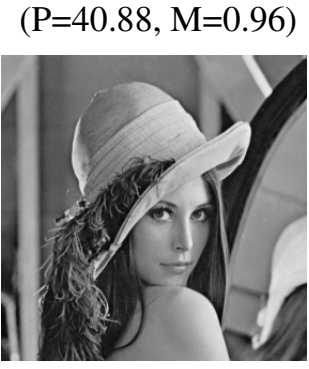

(a)

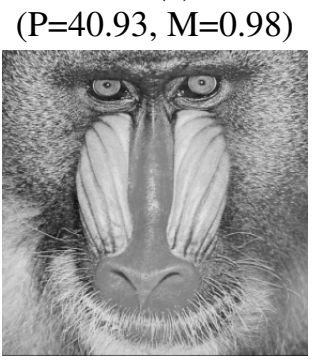

(e)

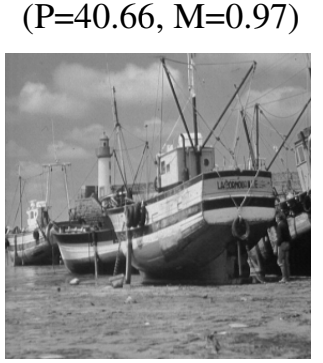

(b)

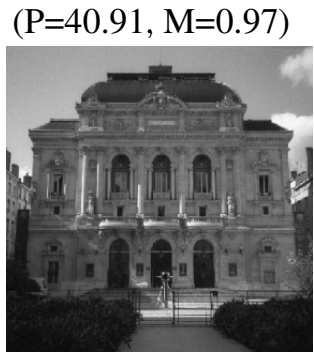

(f)

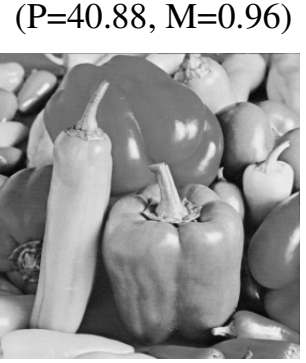

(c)

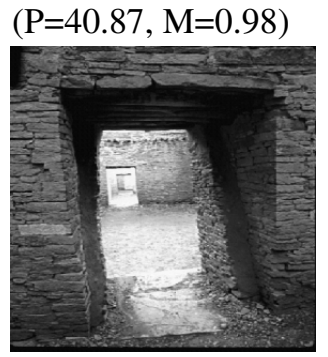

(g)
$(\mathrm{P}=40.77, \mathrm{M}=0.95)$

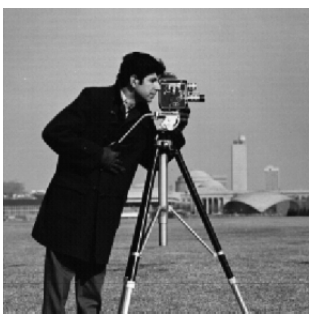

(d)

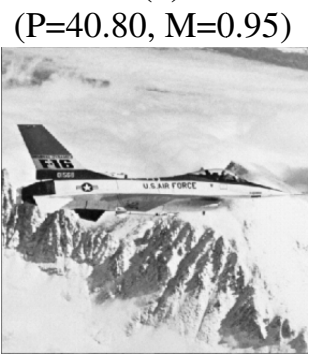

(h)

Figure 8. Watermarked images (a): Lena, (b): Fishing boat, (c): Pepper, (d): Cameraman, (e): Baboon, (f): Opera, (g): Pueblo bonito, (h): F16. (P, M) above each image represents the PSNR (in $\mathrm{dB}$ ) and MSSIM values of the image.

In the second set of experiments, we have studied the performance of the proposed error concealment scheme under different size of lost blocks like $(8 \times 8)$. The lost blocks are simulated in this experimentation by randomly selecting $(8 \times 8)$ blocks and substituting the original pixel values to zero (0). The error blocks are both random and consecutive in nature. The number of lost blocks varies from 50 to 200. Performance results of the proposed error concealment scheme are given in Table 3. Figs. 9(a)-9(d) and Figs. 11(a)-11(d) show the error images having lost blocks of size (8×8). Figs. 10(a)-10(d) and Figs. 12(a)-12(d) are the error concealed images of Figs. 9(a)-9(d), and Figs. 11(a)-11(d), respectively. From Figs. 9-12, it is clear that even if the number of lost blocks reaches 200 , the scheme till can conceals the error effectively. It is already mentioned that we have performed our experiment over large number of images, however, for demonstration purpose we present here result for eight images with varied image characteristics. The numerical values shown in Table 3 indicate that improvement in PSNR (dB) values due to the proposed error concealment method are, on an average, $12 \mathrm{~dB}, 13 \mathrm{~dB}, 13.5 \mathrm{~dB}$ and $14 \mathrm{~dB}$ for the number of lost blocks 50,100, 150, and 200, respectively. The numerical values, in other words, highlight that the proposed error concealment scheme is quite effective even for the large number of lost blocks. Fig. 13 and Fig. 14 show the relative improvement in quality after error concealment in term of PSNR (dB) and MSSIM for different images. Graphical representations reflect the fact that if the image contains high texture regions (like Babon, Pueblo bonito) the error concealment using interpolation, produces poor performance than the images that contain low texture regions likes Lena, Pepper. Figs. 13 and 14 also show that relatively high degree of improvement in quality i.e. PSNR (dB) and MSSIM values are possible to achieve through error concealment, when number of lost blocks is large in number. It is reasonable as less number of lost blocks offers better quality of the received image signal, which in turn does not provide scope of further improvement through error concealment. Moreover, from Figs. 10(c)-10(d) and Figs. 12(c)-12(d), it also clear that when the lost block has a complex geometric structure, such as corner or streaks, our result is not as good at the corner location, as we only consider one edge type in a block. 
Table 3. PSNR (dB) and MSSIM for error concealment with the size of lost block $(8 \times 8)$.

\begin{tabular}{|c|c|c|c|c|c|}
\hline \multirow{2}{*}{\multicolumn{2}{|c|}{ Image }} & \multirow{2}{*}{\multicolumn{2}{|c|}{$\begin{array}{c}\text { PSNR (dB) MSSIM } \\
\text { Before Concealment }\end{array}$}} & PSNR (dB) & MSSIM \\
\hline & & & & \multicolumn{2}{|c|}{ After Concealment } \\
\hline \multirow{4}{*}{ Lena } & 50 & 24.56 & 0.93 & 38.36 & 0.96 \\
\hline & 100 & 22.08 & 0.90 & 36.42 & 0.95 \\
\hline & 150 & 20.41 & 0.87 & 34.88 & 0.94 \\
\hline & 200 & 19.09 & 0.84 & 34.25 & 0.94 \\
\hline \multirow{4}{*}{ Pepper } & 50 & 24.41 & 0.93 & 37.68 & 0.95 \\
\hline & 100 & 21.58 & 0.90 & 35.37 & 0.95 \\
\hline & 150 & 19.72 & 0.87 & 34.10 & 0.94 \\
\hline & 200 & 18.34 & 0.84 & 33.15 & 0.94 \\
\hline \multirow[t]{4}{*}{ Boat } & 50 & 25.11 & 0.94 & 36.28 & 0.96 \\
\hline & 100 & 22.03 & 0.91 & 34.24 & 0.95 \\
\hline & 150 & 20.20 & 0.88 & 33.45 & 0.95 \\
\hline & 200 & 18.69 & 0.85 & 32.06 & 0.94 \\
\hline \multirow[t]{4}{*}{ Baboon } & 50 & 25.36 & 0.96 & 35.55 & 0.98 \\
\hline & 100 & 22.26 & 0.94 & 33.37 & 0.97 \\
\hline & 150 & 20.18 & 0.91 & 31.96 & 0.96 \\
\hline & 200 & 18.82 & 0.88 & 30.64 & 0.95 \\
\hline \multirow[t]{4}{*}{ Opera } & 50 & 22.30 & 0.93 & 40.73 & 0.97 \\
\hline & 100 & 19.40 & 0.89 & 39.56 & 0.96 \\
\hline & 150 & 18.21 & 0.85 & 37.62 & 0.96 \\
\hline & 200 & 17.12 & 0.82 & 36.29 & 0.95 \\
\hline \multirow{4}{*}{ Cameraman } & 50 & 22.41 & 0.91 & 40.73 & 0.95 \\
\hline & 100 & 19.53 & 0.87 & 40.69 & 0.95 \\
\hline & 150 & 17.76 & 0.83 & 39.17 & 0.95 \\
\hline & 200 & 16.67 & 0.79 & 37.65 & 0.95 \\
\hline \multirow[t]{4}{*}{ Pueblo bonito } & 50 & 28.43 & 0.96 & 36.88 & 0.97 \\
\hline & 100 & 25.94 & 0.94 & 34.72 & 0.97 \\
\hline & 150 & 24.51 & 0.92 & 33.97 & 0.96 \\
\hline & 200 & 23.83 & 0.90 & 33.34 & 0.96 \\
\hline \multirow[t]{4}{*}{ F16 } & 50 & 21.93 & 0.91 & 40.32 & 0.95 \\
\hline & 100 & 18.82 & 0.87 & 39.49 & 0.95 \\
\hline & 150 & 16.88 & 0.83 & 39.25 & 0.94 \\
\hline & 200 & 15.53 & 0.79 & 39.17 & 0.94 \\
\hline
\end{tabular}

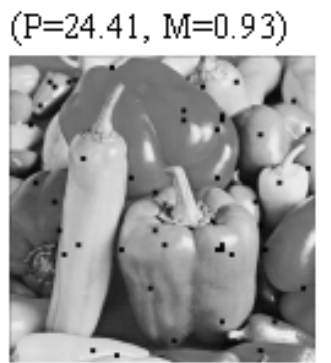

(a)

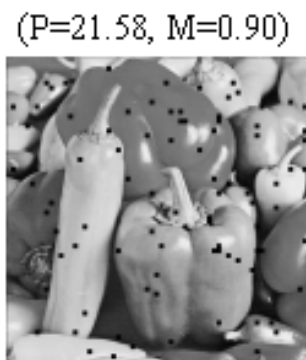

(b)

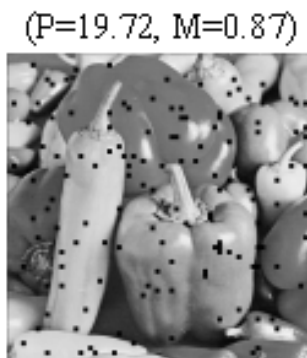

(c)

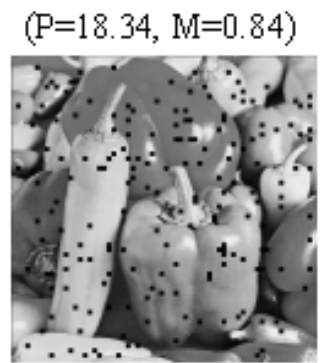

(d)

Figure 9. Error image Pepper: (a) 50 lost blocks; (b) 100 lost blocks; (c) 150 lost blocks; (d) 200 lost blocks. 


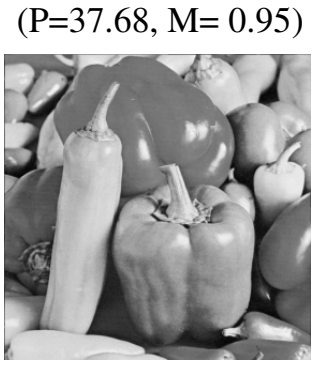

(a)

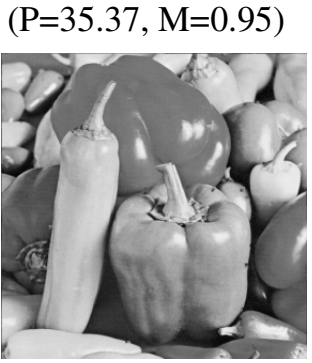

(b)

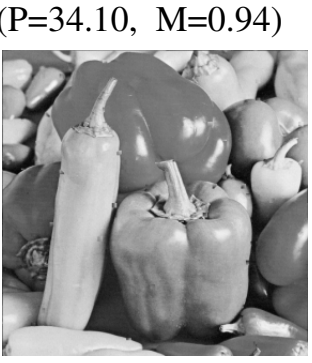

(c)

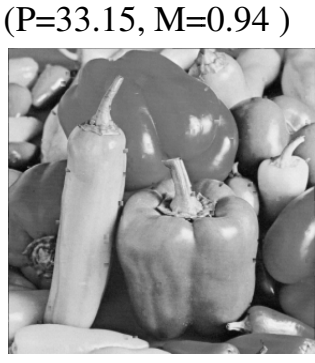

(d)

Figure 10. Error concealed image Pepper: (a) 50 loss blocks; (b) 100 loss blocks; (c) 150 loss blocks; (d) 200 loss blocks.

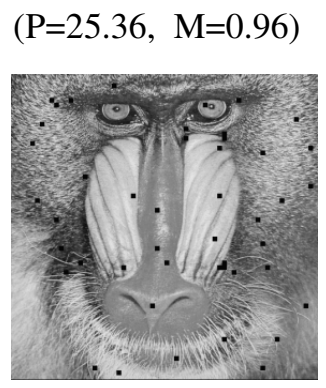

(a)

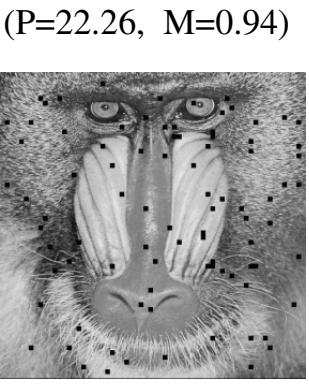

(b)

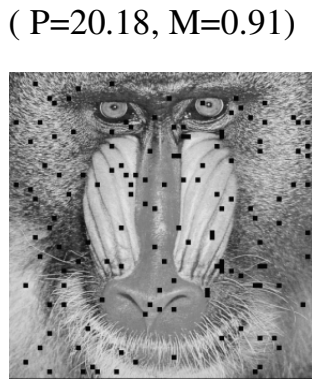

(c)
$(\mathrm{P}=18.82, \mathrm{M}=0.88)$

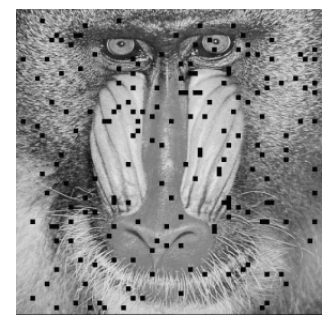

(d)

Figure 11. Error image Baboon: (a) 50 lost blocks; (b) 100 lost blocks; (c) 150 lost blocks; (d) 200 lost blocks.

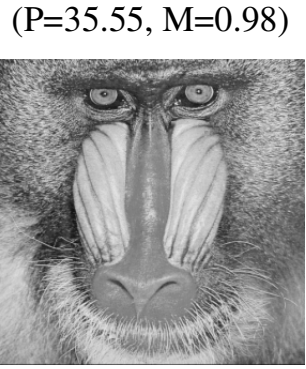

(a)

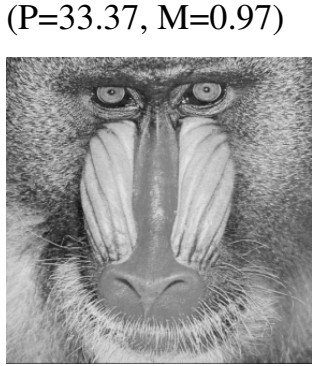

(b)

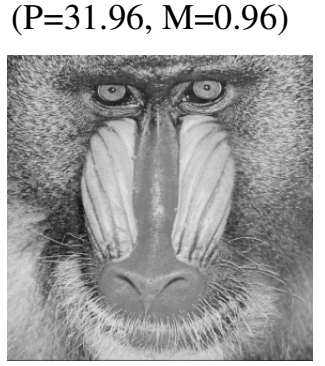

(c)

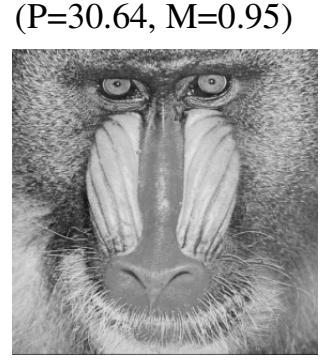

(d)

Figure 12. Error concealed image Baboon: (a) 50 lost blocks; (b) 100 lost blocks; (c) 150 lost blocks; (d) 200 lost blocks.

The experimental results shown in Fig. 13 and Fig. 14 also suggest that the proposed error concealment scheme is quite effective for slow fading as well as for the wireless channel suffered by deep fades. In our scheme, less number of lost blocks is considered as the channel is under low fades, while large number of lost blocks is considered as a deep fades. The algorithm developed can be made use to design an adaptive transmission scheme through the estimation of channel parameters (embedded edge information that acts as pilot signal without effecting bandwidth and synchronization problem like the existing pilot based system) as well as image and video signal transmission over deeply faded channel. The quality of the extracted edge information will indicate the current status of the channel condition. When the quality of the error concealed image goes below the acceptable level, feedback information from the receiver to the transmitter to be sent indicating that the channel is under deep fade and current data rate is 
not well suited. The data transmission rate may be lowered for sometimes so long the quality of the error concealed image goes above the acceptable threshold level.

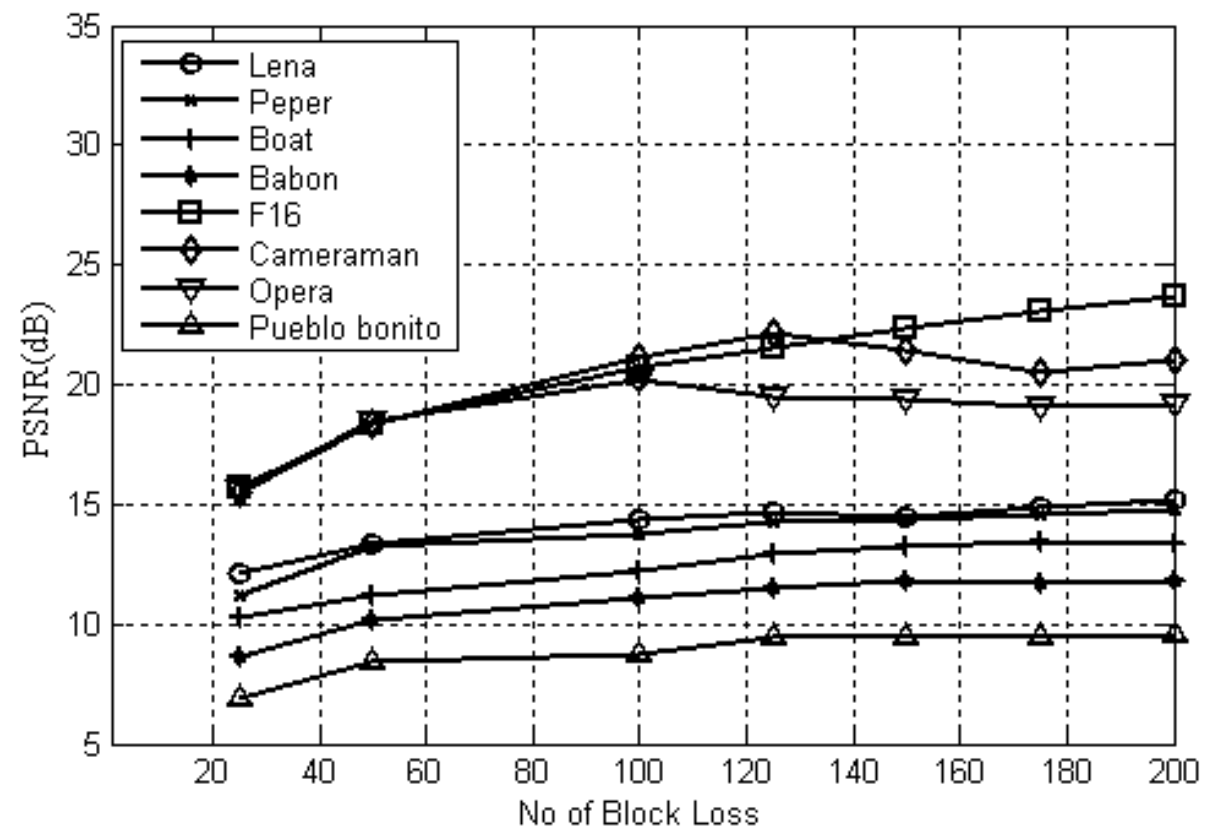

Figure 13. Improvement in quality after error concealment in term of PSNR (dB).

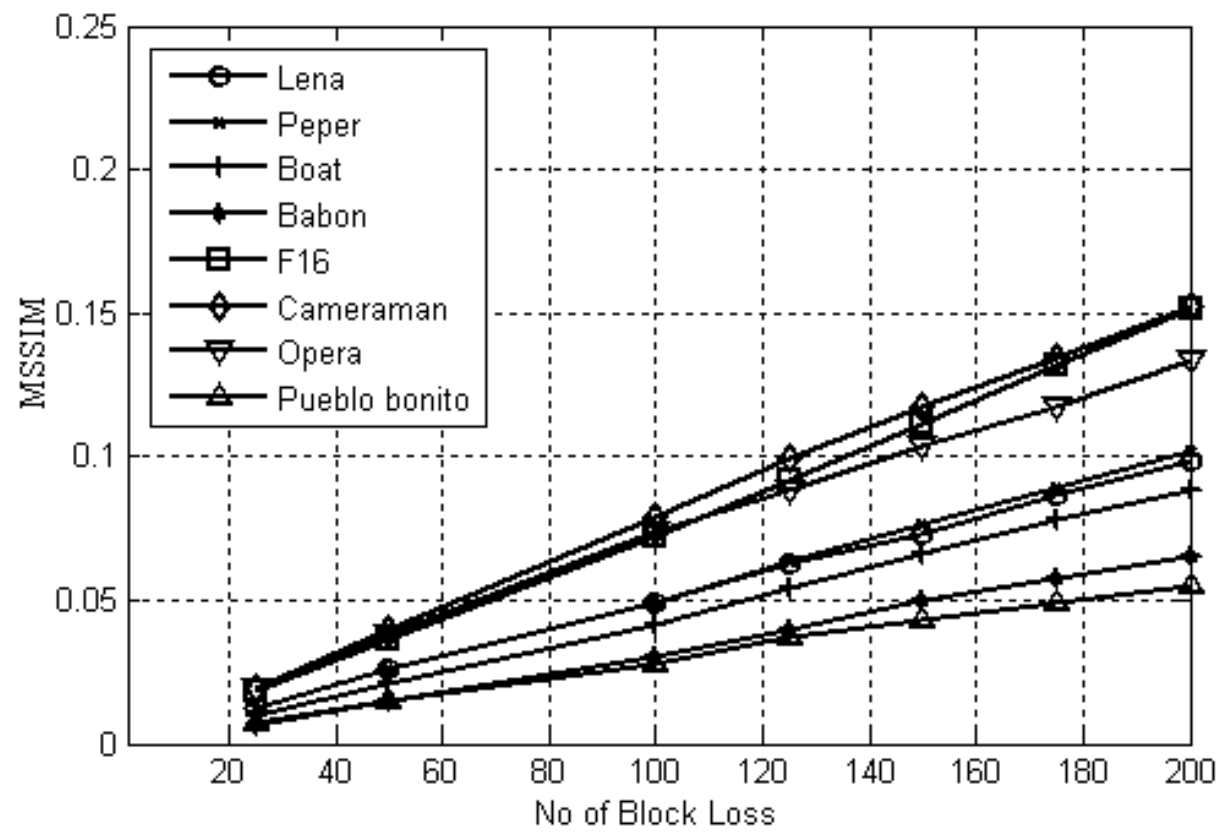

Figure 14. Improvement in quality after error concealment in term of MSSIM.

In the third set of experiments, we have studied the effect of different watermark power (WP) on the relative gain/loss in performance for error concealment. The watermark power is defined as [23]

$$
W P=10 \log _{10} \frac{\Delta^{2}}{12} \mathrm{~dB}
$$


where the symbol $\Delta$ corresponds to the step size used for watermarking. Table 4 shows the relative visual quality of the watermarked images for different watermark power obtained through different step sizes. The corresponding MSSIM values are also reported in order to have an idea about the loss in structural information due to the variation of watermark powers. Fig. 15 and Fig. 16 show the relative improvement in quality after error concealment with respect to PSNR (dB) and MSSIM for different watermark power. Graphical representations reflect the fact that when watermark embedding power is increased, the improvement in both PSNR $(\mathrm{dB})$ and MSSIM values are decreased. It is also interesting to see from the results of Fig. 15 and 16 that although for a given watermark power, the relative improvement in visual quality in PSNR (dB) does not vary much for the number of loss blocks varying from 50 to 200 but significant improvement in structural information is found with the loss of more number of blocks.

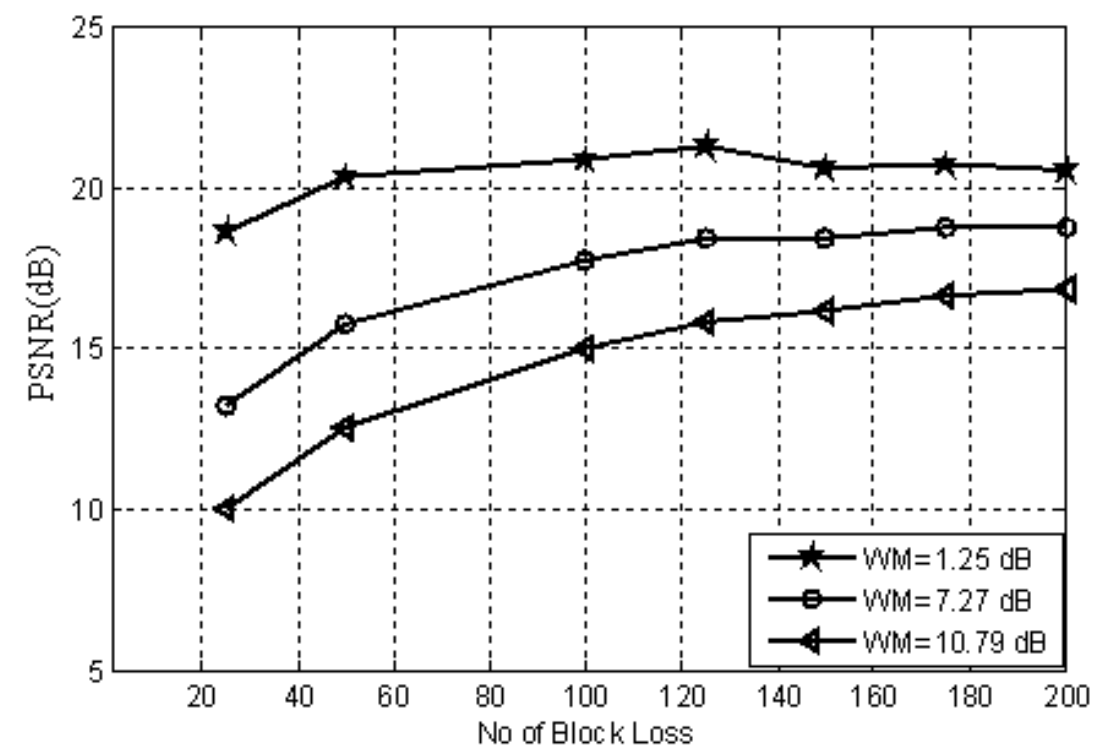

Figure 15. Improvement in PSNR (dB) after concealment of lost block for different WP.

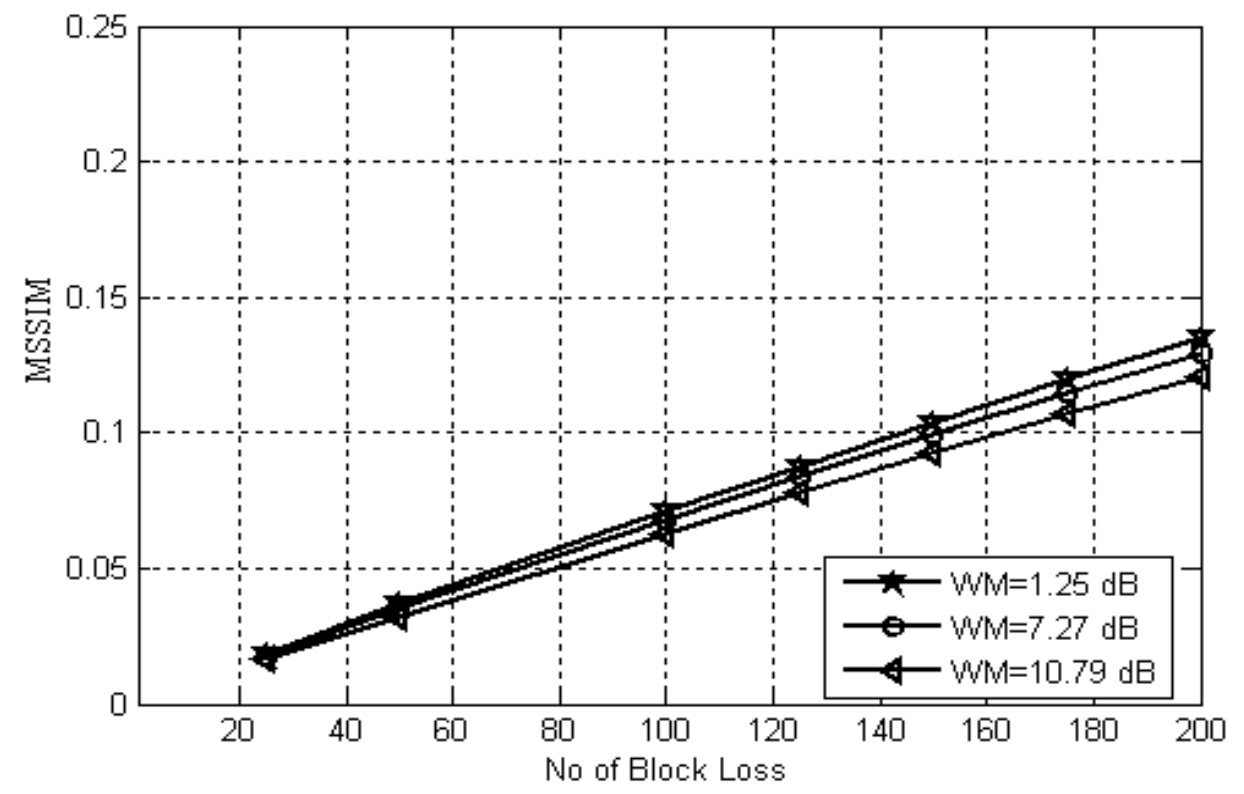

Figure 16. Improvement in MSSIM after error concealment of lost block for different WP. 
Table 4. Average PSNR (dB) and MSSIM values for watermarked images with different watermark powers.

\begin{tabular}{|l|l|l|l|}
\hline WP(dB) & $\mathbf{1 . 2 5}$ & $\mathbf{7 . 2 7}$ & $\mathbf{1 0 . 7 9}$ \\
\hline Step size $(\triangle)$ & 4 & 8 & 12 \\
\hline PSNR $(\mathrm{dB})$ & 46.94 & 40.88 & 37.28 \\
\hline MSSIM & 0.99 & 0.96 & 0.92 \\
\hline
\end{tabular}

Table 5. NCC values for various image processing operations and geometric attacks.

\begin{tabular}{|c|c|c|c|c|c|c|c|c|}
\hline $\begin{array}{c}\text { Image } \\
\text { processing } \\
\text { operations }\end{array}$ & $\begin{array}{c}\text { JPEG } \\
\mathbf{9 5}\end{array}$ & $\begin{array}{c}\text { JPEG } \\
\mathbf{8 5}\end{array}$ & $\begin{array}{c}\text { JPEG } \\
\mathbf{7 5}\end{array}$ & $\begin{array}{c}\text { JPEG } \\
\mathbf{7 0}\end{array}$ & $\begin{array}{c}\text { Mean } \\
\text { Filtering } \\
\mathbf{( 3 \times 3 )}\end{array}$ & $\begin{array}{c}\text { Median } \\
\text { Filtering } \\
\mathbf{( 3 \times 3 )}\end{array}$ & $\begin{array}{c}\text { Down } \\
\mathbf{8} \text { Up } \\
\text { Sampling } \\
\mathbf{( 0 . 7 5 )}\end{array}$ & $\begin{array}{c}\text { Rotation } \\
\mathbf{( 0 . 2 5 )}\end{array}$ \\
\hline $\begin{array}{c}\text { Without } \\
\text { M-ary } \\
\text { QIM }\end{array}$ & 0.91 & 0.73 & 0.61 & 0.53 & 0.82 & 0.86 & 0.93 & 1.00 \\
\hline $\begin{array}{c}\text { M-ary } \\
\text { QIM }\end{array}$ & 0.99 & 0.99 & 0.99 & 0.99 & 0.95 & 0.96 & 0.98 & 1.00 \\
\hline
\end{tabular}

In the fourth set of experiments, we have studied the robustness performance of the proposed scheme for common image and signal processing operations and geometric attacks like slight rotation available in benchmark StirMark 4 [24, 25, 26], since QIM-based scheme is usually bristle for those operations. For image scaling operation, before watermark extraction, the attacked image is rescaled to the original size. For rotation operation, we have estimated the rotation angle of the watermarked images by control point selection method with the help of the original images. The rotated watermarked images are then inverse rotated and corrected by linear interpolation. Now, those corrected watermarked images are used for watermark detection. This is done to compensate the effect of lost in data due to the rotation operation. Table 5 shows the performance improvement in term of normalized cross-correlation (NCC) [27] if three watermark bits (that are used to represent the edge information) are inserted in a $(8 \times 8)$ blocks using traditional QIM and M-ary QIM. It is seen that M-ary modulation offers higher robustness over traditional QIM that ultimately helps in extracting efficient edge information for the concealment purpose.

The computational complexity of the decoder in terms of required number of additions, subtraction, division and multiplications is calculated and is also compared with other methods $[4,28]$. Table 6 summarizes the comparison results for restoring 64 pixel values i.e. one $(8 \times 8)$ block. Generally, edge-based methods [4] are computationally intensive since they require estimation of edge directions. On the other hand, the scheme proposed in [28] returns high complexity as the scheme is based on solving a set of 8 linear equations with 8 unknowns. The result in Table 6 highlights the superiority of our method over $[4,28]$.

Table 6. Computational complexity of various error concealment methods.

\begin{tabular}{|c|c|c|c|c|c|}
\hline Method & Addition & Subtraction & Multiplication & Division & Total \\
\hline Method [28] & 1720 & - & 1920 & - & 3640 \\
\hline Method [4] & 8256 & & 4096 & & 12,352 \\
\hline Proposed Method & 315 & 315 & - & 315 & 945 \\
\hline
\end{tabular}


Table 7. Comparison between the proposed and reference methods.

\begin{tabular}{|l|l|l|l|l|l|l|l|}
\hline PLR & $\mathbf{3 \%}$ & $\mathbf{5 \%}$ & $\mathbf{1 0 \%}$ & $\mathbf{1 5 \%}$ & $\mathbf{2 0 \%}$ & $\mathbf{2 5 \%}$ & $\mathbf{3 0 \%}$ \\
\hline Lee et al. (2004) [33] & 31.12 & 26.22 & 25.04 & 24.14 & 23.16 & - & - \\
\hline Lie et al. (2005) [31] & 35.62 & 34.98 & 33.34 & 32.43 & 31.35 & - & - \\
\hline Gur et al. (2005) [13] & 28.91 & 27.72 & 26.10 & 25.21 & 24.43 & 23.98 & 23.85 \\
\hline Chen et al. (2006)[2] & - & 28.72 & 26.12 & 24.47 & - & - & - \\
\hline Lin et al. (2008) [29] & - & 40.91 & 36.87 & 35.02 & 34.08 & 33.09 & 31.72 \\
\hline Anhari et al. (2008) [30] & 37.45 & 36.12 & 34.82 & 34.40 & 31.90 & 30.91 & 30.13 \\
\hline Wu et al. (2008) [32] & 34.07 & 28.43 & 27.11 & 25.16 & 24.98 & - & - \\
\hline Ma et al. (2009) [4] & 35.51 & 34.00 & 28.12 & 26.57 & 24.10 & - & - \\
\hline Yýlmaz et al. [15] & 38.12 & 35.17 & 33.53 & 27.17 & 28.14 & 29.65 & 29.24 \\
\hline Yin et al. [16] & 39.81 & 38.54 & 36.15 & 32.10 & 33.57 & 32.32 & 32.10 \\
\hline Proposed & 39.95 & 39.83 & 38.69 & 38.42 & 37.15 & 35.12 & 33.52 \\
\hline
\end{tabular}

Error reconstruction performance of the proposed method is compared with both non-data hiding $[2,4,32,33]$ and data hiding based methods [13, 15, 16, 29, 30, 31]. It is observed from the results shown in Table 7 that our method offers better PSNR (dB) values even if packet loss rate (PLR) increases to 30\%. From Table 7 it is clear that when PLR is low, non-data hiding based error concealment methods offer similar performance like data hiding based approaches. On the other hand, the former (non-data hiding based) methods result low improvement in quality at high burst error condition. This is because the methods [2, 4, 32, 33] recover error blocks depending on the correlation/interpolation of the received surrounding blocks in the decoder. As a matter of fact, those schemes work well in simplified loss scenarios where successfully received data are assumed to be reconstructed loss-free. This is often not the case. Hence, those schemes return low improvement in quality at high burst error condition (i.e. at high PLR), as most of the surrounding blocks may be lost. We also compare the result of the proposed method with the work reported in $[13,15,16,29,30,31]$. Results shown in Table 7 highlight the superiority of our method. The schemes proposed in $[15,16]$ offer poor performance as the data (edge information) embedding is done using even/odd embedding. The even/odd embedding is fragile in nature. The embedded data may be lost due to signal attenuation. In our scheme, both the above drawbacks are eliminated using M-ary QIM data hiding and conceal the error quite effectively in burst error condition, even though there is some quality degradation due to data embedding at the transmitter. Moreover, it is also seen that the use of data hiding reduces nearly $32.05 \%$ computation time of the decoder over [2,4]. The test is conducted on Pentium IV, $2.80 \mathrm{GHz}$ processor, with $512 \mathrm{MB}$ RAM using MATLAB 7 version and when $50 \%$ of $(8 \times 8)$ blocks are erroneous.

\section{CONCLUSION}

An image error concealment scheme based on M-ary QIM watermarking is proposed. The capacity of data hiding is increased with the help of M-ary modulation. The scheme reduces decoder workload (computation power) nearly about $32.05 \%$, which makes the scheme suitable for real time error concealment where the decoder may not have sufficient computation power or implementation done in online. Simulation results also demonstrate that the proposed scheme provides significant improvement in term of objective evaluations especially for high burst error condition. Finally, the proposed error concealment scheme can be made use for design of an adaptive data transmission scheme through the estimation of wireless channel condition using extracted edge direction information. Future work can be concentrated on performance improvement of the proposed scheme using channel coding and as well as development of hardware implementation of the proposed scheme. 


\section{REFERENCES}

[1] Sklar, B., (1997) "Rayleigh fading channels in mobile digital communication systems, part I: Characterization”, IEEE Communication Magazine, Vol. 35, No. 9, pp 90-100.

[2] Chen, B. N., \& Lin Y, (2006) "Hybrid error concealment using linear interpolation", In Proc. International Symposium on Communications and Information Technologies, Bangkok, pp 926 931.

[3] Nemethova, O., Moghrabi, A. A., \& Rupp M, (2005) "Flexible error concealment for H.264 based on directional interpolation", In Proc. International Conference on Wireless Networks, Communications and Mobile Computing, Maui, HI, pp 1255 - 1260.

[4] Ma, M., Au, O. C., Chan, S. H. G., \& Sun M. T., (2009) "Edge-directed error concealment", IEEE Transaction on Circuits and Systems for Video Technology, doi 10.1109/TCSVT.2009.2035839.

[5] Sun, H., \& Kwok W., (1995) "Concealment of damaged block transform coded images using projection onto convex sets”, IEEE Transaction on Image Processing, Vol. 4, No. 4, pp 470477.

[6] Chen, M. J., Chen, C. S., \& Chi M. C., (2003) "Recursive block-matching principle for error concealment algorithm", In Proc. of International Symposium on Circuits and Systems, Iasi, Romania, pp $528-531$.

[7] Wong, W. Y. F., Cheng, A. K. Y., \& Horace H. S., (2001) "Concealment of damaged blocks by neighbourhood regions partitioned matching", In Proc. of International Conference on Image Processing, Thessaloniki, Greece, pp 45-48.

[8] Cao, J., Li, F., \& Guo J., (2003) “An efficient error concealment algorithm for DCT encoded images", In Proc. IEEE Canadian Conference on Electrical and Computer Engineering, Canada, pp $753-756$.

[9] Rane, S. D., Remus, J., \& Sapiro G., (2002) "Wavelet-domain reconstruction of lost blocks in wireless image transmission and packet-switched networks", In Proc. International Conference on Image Processing, Rochester, New York, pp 309-312.

[10] Li, X., \& Orchard M. T., (2002) "Novel sequential error-concealment techniques using orientation adaptive interpolation”, IEEE Transaction on Circuits System Video Technology, Vol. 12, No. 10, pp 857-864.

[11] Liu, Y., \& Li Y., (2000) "Error concealment for digital images using data hiding", In Proc. of the Ninth DSP Workshop, pp 1-6.

[12] Adsumilli, C. B., Farias, M. C. Q., Mitra, S. K., \& Carli M., (2005) “A robust error concealment technique using data hiding for image and video transmission over lossy channels", IEEE Transactions on Circuits and Systems for Video Technology, Vol. 15, No. 11, pp 1394-1406.

[13] Gur, G., Alagoz, F., \& AbdelHafez M., (2005) “A novel error concealment method for images using watermarking in error-prone channels", In Proc. $16^{\text {th }}$ IEEE International Symposium on Personal, Indoor and Mobile Radio Communications, Germany, pp 2637 - 2641.

[14] Phadikar, A., \& Maity S. P., (2009) "Image error concealment based on data hiding using dualtree complex wavelets," In Proc. of the $4^{\text {th }}$ Indian International Conference of Artificial Intelligence, Tumkur, India, pp 1764-1781.

[15] Yýlmaz, A., \& Alatan A. A., (2003) "Error concealment of video sequences by data hiding", In Proc. International Conference on Image Processing, Barcelona, Spain, pp 679-82.

[16] Yin, P., Liu, B., \& Yu H. H., (2001) "Error concealment using data hiding", In Proc IEEE International Conference on Acoustics, Speech, and Signal Processing, Salt Lake City, Utah, pp $1453-1456$.
C h e n
B . ,
$\begin{array}{llllllll}\& & \mathrm{~W} & \mathrm{O} & \mathrm{r} & \mathrm{n} & \mathrm{e} & \mathrm{l} & 1\end{array}$
$\left.\begin{array}{lllllll}1 & & & & G & \\ ( & 2 & 0 & 0 & 1 & \end{array}\right)$ 


\begin{tabular}{|c|c|c|c|c|c|c|c|c|c|c|c|c|c|c|c|c|c|c|c|}
\hline $\mathrm{m}$ & $\mathrm{O}$ & $\mathrm{d}$ & $\mathrm{u}$ & 1 & $\mathrm{a}$ & $\mathrm{t}$ & $\mathrm{i}$ & $\mathrm{O}$ & $\mathrm{n}$ & : & & & $\mathrm{c}$ & 1 & $\mathrm{a}$ & S & S & & o \\
\hline $\mathrm{p}$ & $\mathrm{r}$ & $\mathrm{O}$ & V & $\mathrm{a}$ & $\mathrm{b}$ & 1 & $\mathrm{y}$ & & $\mathrm{g}$ & O & $\mathrm{O}$ & $\mathrm{d}$ & & $\mathrm{m}$ & $\mathrm{e}$ & $\mathrm{t}$ & $\mathrm{h}$ & o & $\mathrm{d}$ \\
\hline$f$ & $\mathrm{O}$ & $\mathrm{r}$ & & & & & & & & & & & & $\mathrm{d}$ & $\mathrm{i}$ & $\mathrm{g}$ & $\mathrm{i}$ & $\mathrm{t}$ & $\mathrm{a}$ \\
\hline y & $\mathrm{a}$ & $\mathrm{t}$ & $\mathrm{e}$ & $\mathrm{r}$ & $\mathrm{m}$ & $\mathrm{a}$ & $\mathrm{r}$ & $\mathrm{k}$ & $\mathrm{i}$ & $\mathrm{n}$ & $\mathrm{g}$ & & & & & & & $\mathrm{a}$ & $\mathrm{n}$ \\
\hline
\end{tabular}

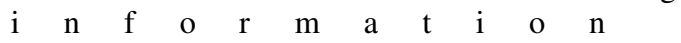

$\mathrm{e} \mathrm{m} \quad \mathrm{b}$ e $\mathrm{d} \quad \mathrm{d} \quad \mathrm{i} \quad \mathrm{n} \quad \mathrm{g} \quad, \quad$ IEEE Transaction of Information Theory, Vol. 47, pp 1423-1443.

[18] Gonzalez, R. C., Woods, R. E., \& Eddins S. L., (2005) Digital image processing using Matlab, Pearson Education.

[19] Maity, S. P., Kundu, M. K., Das, T. S., \& Nandi P. K., (2005) "Robustness improvement in spread spectrum watermarking using M-ary modulation", In Proc. 11th National Conf. on Communication, India, pp 569-573.

[20] Ngan, K. N., \& Stelle R., (1982) "Enhancement of PCM and DPCM images corrupted by transmission errors", IEEE Transaction on Communication, Vol. 30, pp 257-265.

[21] http://www.petitcolas.net/fabien/watermarking/image_database/index.ht ml.

[22] Wang, Z., Bovik, A. C., Sheikh, H. R., \& Simoncelli E. P., (2004) "Image quality assessment: from error measurement to structural similarity”, IEEE Transactions on Image Processing, Vol. 3 , pp 1-14.

[23] Boyer, J. P., Duhamel, P., \& Talon J. B. (2006) "Performance analysis of scalar DC-QIM for watermark detection," In Proc. of IEEE International Conference on Acoustics, Speech and Signal Processing, Honolulu, Toulouse, pp 237- 240.

[24] Petitcolas, F. A. P., (2000) "Watermarking schemes evaluation", IEEE Signal Processing, Vol. 17, pp 58-64.

[25] Petitcolas, F. A. P., Anderson, R. J., \& Kuhn M. G., (1998) “Attacks on copyright marking systems”, In Proc. of Second International Workshop on Information Hiding (LNCS 1525), Springer-Verlag, pp 219-239.

[26] (StirMark $4.0 \quad$ Online Available: <http://www.petitcolas.net/fabien/watermarking/stirmark/>.

[27] Phadikar, A., Maity, S. P., \& Mandal M. K., (2008) "Quantization based data hiding scheme for quality access control of images," In Proc. of the $12^{\text {th }}$ IASTED International Conference on Internet and Multimedia Systems and Applications (IMSA 2008), Hawaii, USA, pp 113-118.

[28] Shirani, S., Kossentini, F., \& Ward R., (2000) "Reconstruction of baseline JPEG coded images in error prone environments", IEEE Transactions on Image Processing, Vol. 9, No. 7, pp 12921298.

[29] Lin, S. D., \& Tsai C. W., (2008) “An effective data embedding for error concealment", In Proc. $3 r d$ International Conference on Innovative Computing Information and Control, Dalian, pp 2832.

[30] Anhari, A. K., Sodagari, S. , \& Avanaki A. N., (2008) "Hybrid error concealment in image communication using data hiding and spatial redundancy," In Proc. International Conference on Telecommunications, pp $1-5$.

[31] Lie, W. N., Lin, T. C. I., Tsai, D. C., \& Lin G. S., (2005) "Error resilient coding based on reversible data embedding technique for H.264/AVC video", In Proc. International Conference on Multimedia and Expo, Amsterdam, Netherlands, pp 1174 - 1177.

[32] Wu, J., Liu, X., \& Yoo K. Y. (2008) "A temporal error concealment method for H.264/AVC using motion vector recovery", IEEE Transaction on Consumer Electronics, Vol. 54, No. 4, pp 1880-1885.

[33] Lee, P. J., Chen, H. H., \& Chen L. G., (2004) "A new error concealment algorithm for H.264/AVC video transmission”, In Proc. International Symposium On Intelligent Multimedia, Video, and Speech Processing, Hong Kong, pp 619-622. 


\section{Authors}

Amit Phadikar received his B.E. in Computer Sc. and Engineering in 2002 from Vidyasagar University, W.B., India and M.Tech degree in Information Technology (Artificial Intelligence) in 2005 from Rajiv Gandhi Proudyogiki Vishwavidyalaya (RGPV), Bhopal, M.P., India. Since 2005 he is working as Lecturer at the Department of Information Technology, MCKV Institute of Engineering, Liluha, Howrah, W.B., India.

His research areas include digital image watermarking, digital signal processing, content-based image retrieval. Currently he is working towards his Ph.D. degree in Engineering (Information Technology) from Bengal Engineering and Science University, Shibpur, India.

Santi P. Maity received his B.E. in Electronics and Communication Engineering and M.Tech degree in Microwaves, both from the University of Burdwan, India. He received his Ph.D. degree in Engineering (Computer Science and Technology) from Bengal Engineering and Science University, Shibpur, India. During January 2009 to July, 2009 he did his pos-doctoral work concerning watermarking in lured applications in the "Laboratoire des Signaux et Systems (CNRS-Supelec-Universite Paris-Sud 11)" in France. He is at present working as Associate Professor at the Department of Information Technology, Bengal Engineering and Science University, Shibpur.

His research areas include digital image watermarking, multiuser detection in CDMA, digital signal processing, digital wireless communication, VLSI watermarking. He has contributed about 80 research papers in well-known and prestigious archival journals, international refereed conferences and as chapters in edited volumes.
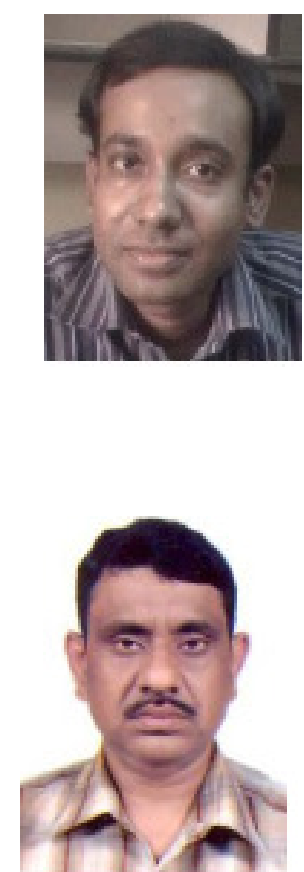